\title{
TRANSIENT AND STEADY-STATE ABSORPTIONS OF A WEAK PROBE FIELD IN A COUPLED DOUBLE QUANTUM-WELL STRUCTURE
}

\author{
WEN-XING YANG*,†,, JIN XU* $^{*}$ and RAY-KUANG LEE ${ }^{\dagger}$ \\ * Department of Physics, Southeast University, Nanjing 210096, China \\ $\dagger$ Institute of Photonics Technologies, National Tsing Hua University, Hsinchu 300, Taiwan \\ ¥wenxingyang@seu.edu.cn
}

Received 9 November 2008

\begin{abstract}
We propose and analyze an efficient scheme for suppressing the absorption of a weak probe field based on intersubband transitions in a four-level asymmetric coupled quantum well (CQW) driven coherently by a probe laser field and a control laser field. We study the steady-state process analytically and numerically, and our results show that the probe absorption can be completely eliminated under the condition of Raman resonance (i.e. two-photon detuning is zero). Besides, we can observe one transparency window without requiring one- or two-photon detuning to exactly vanish. This investigation may provide a possible scheme for EIT in solids by using the CQW.
\end{abstract}

Keywords: Transient absorption; steady state; quantum well structure.

PACS Number(s): 42.50.Gy, 78.67.De

\section{Introduction}

The optical properties of cold atomic gases can be radically modified by laser beams. ${ }^{1}$ A strong laser light can essentially modify atomic states through the quantum interference between excitation pathways. Under the conditions of electromagnetically induced transparency (EIT), ${ }^{2}$ it is possible to control the optical response and related absorption of weak laser light. This effect has been deeply studied in atomic physics, ${ }^{1-10}$ starting from its observation in sodium vapors, ${ }^{11}$ and has disclosed new possibilities for nonlinear optics and quantum information processing. For example, it has been demonstrated that EIT can be used to suppress both single-photon and two-photon absorptions in the three-level atomic medium. ${ }^{6}$ Schemes to suppress absorptions of the short-wavelength light based on EIT have also been proposed. ${ }^{9}$ In particular, few authors analyzed and discussed the suppression of both two- and three-photon absorptions in four-wave mixing (FWM) and hyper-Raman scattering (HRS) in the resonant coherent atomic medium via EIT. ${ }^{7-9}$

As we know, it is easy to implement EIT in optically dense atomic media in the gas phase, but more difficult to observe EIT in the solid-state media due to 
the short coherence times in the solid-state system. ${ }^{1}$ Nevertheless, some investigations have demonstrated EIT effect and ultraslow optical pulse propagation in semiconductor quantum well (QW) structure. ${ }^{12-18}$ For example, Sadeghi et al. ${ }^{13}$ in an asymmetric QW, showed that EIT may be obtained with appropriate driving fields, provided that the coherence between the intersubbands considered is preserved for a sufficiently long time. So, quantum coherence and interference in QW structures have attracted great interest due to the potentially important applications in optoelectronics and solid-state quantum information science. In fact, except for the investigations of EIT, Autler-Townes splitting, ${ }^{18}$ gain without inversion, ${ }^{19}$ modified Rabi oscillations and controlled population transfer, ${ }^{20,21}$ largely enhanced second harmonic generation, ${ }^{22}$ controlled optical bistability, ${ }^{23}$ ultrafast all-optical switching, ${ }^{24}$ phase-controlled behavior of absorption and dispersion, ${ }^{25}$ coherent population trapping, ${ }^{26}$ enhanced refraction index without absorption, ${ }^{27}$ FWM,${ }^{28}$ and several other phenomena, ${ }^{29-31}$ have been theoretically studied and experimentally observed in the intersubband transitions (ISBTs) of semiconductor QWs.

In this work, we take note of the calculation of the model for an asymmetric semiconductor coupled double $\mathrm{QW},{ }^{32-35}$ in which the realization of a photon switch and the optical bistability have been studied based on quantum interference between ISBTs in this system. ${ }^{33}$ In contrast to previous work based on Fano-type interference transitions, in this study we focus on the absorptions of a weak probe field based on the conditions of Raman resonance, and analyze the probe absorption properties for the transient- and steady-state processes in such systems. Different from the ISBTs in the infrared region, we consider such transition in the ultraviolet or visible region, in which it has a very large dipole moment length, and so the optoelectronic devices may be realized with a smaller pulse intensity. Besides, devices based on semiconductor QW structures have many inherent advantages, such as large electric dipole moments due to the small effective electron mass, high nonlinear optical coefficients, and great flexibility in device design by choosing the materials and structure dimensions. As a result, our investigations also provide a robust transparency window without requiring that the one- or two-photon detuning be exactly zero.

\section{The Physical Model and Equations of Motion}

Let us consider the asymmetric semiconductor coupled double QW structure consisting of 10 pairs of a 51-monolayer $(145 \AA)$ thick wide well and a 35-monolayer (100 ̊) thick narrow well, separated by an $\mathrm{Al}_{0.2} \mathrm{Ga}_{0.8} \mathrm{As}$ buffer layer, ${ }^{34,35}$ as shown in Fig. 1. In this QW structure, the first $(n=1)$ electron level in the wide well and in the narrow well can be energetically aligned with each other by applying a static electric field, while the corresponding $n=1$ hole levels are never aligned for this polarity of the field. For transitions from the wide well and the narrow well, the Coulomb interaction between the electron and the hole downshifts the value of the 


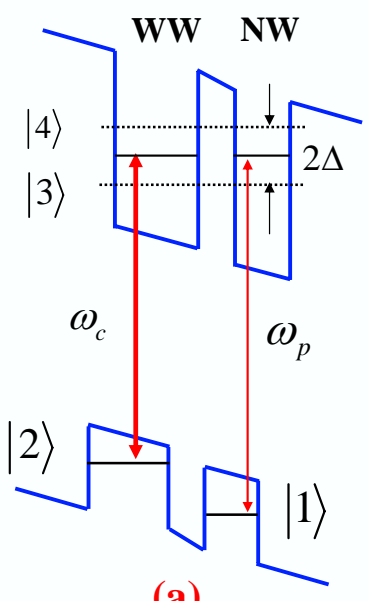

(a)

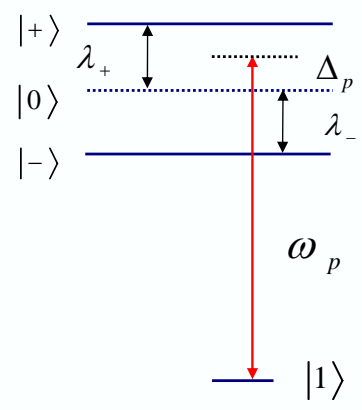

(b)

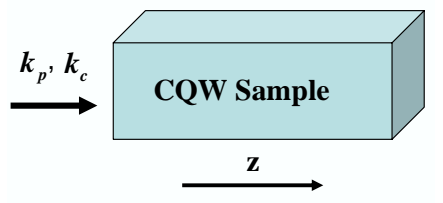

(c)

Fig. 1. Conduction subband energy level diagram for an asymmetric coupled QWs consisting of a wide well (WW) and a narrow well (NW), as shown in Ref. 34. (a) Levels $|3\rangle$ and $|4\rangle$ are the bonding and the antibonding electron state of the coupled system and Levels $|1\rangle$ and $|2\rangle$ are the localized hole states. $2 \Delta, \omega_{c}$ and $\omega_{p}$ are the energy splitting between tunnel-coupled electronic levels, and the frequency of the strong coupled field and of the weak probe field, respectively. (b) Corresponding to the dressed-state picture for the case $\Delta_{c}=0$ : subband $|2\rangle$ couples with subbands $|3\rangle$ and $|4\rangle$ simultaneously giving rise to the three dressed states $|+\rangle,|0\rangle$ and $|-\rangle$. (c) All the lights propagate along the $z$ axis within our CQW sample.

electric field where the resonance condition is fulfilled corresponding to the built-in field. Level $|3\rangle$ and level $|4\rangle$, corresponding respectively to the bonding state and the anti-bonding state, are created by tunnel-coupling the first $(n=1)$ electron level in the wide well and in the narrow well. The energy difference, $2 \Delta$, between the bonding state and the antibonding state is determined by the level splitting in the absence of tunneling and the tunneling matrix element, and can be controlled by an electric field applied perpendicularly to coupled QWs. Level $|1\rangle$ in the narrow well and level $|2\rangle$ in the wide well are localized hole states.

We assume that the transitions $|2\rangle \leftrightarrow|4\rangle$ and $|2\rangle \leftrightarrow|3\rangle$ are simultaneously driven by a strong coupling field with the respective one-half Rabi frequencies $\Omega_{c}=\mu_{42} E_{c} / 2 \hbar$ and $\Omega_{c} \mu_{32} / \mu_{42}$. At the same time, a weak probe field is applied to the transitions $|1\rangle \leftrightarrow|4\rangle$ and $|1\rangle \leftrightarrow|3\rangle$ simultaneously with the respective Rabi frequencies $\Omega_{p}=\mu_{41} E_{p} / 2 \hbar$ and $\Omega_{p} \mu_{31} / \mu_{41} . E_{c}$ and $E_{p}$ are the amplitude of the strong-coupling field and of the weak probe field, respectively. $\mu_{i j}=\mu_{i j} \cdot \widehat{e}_{L}$ is 
the dipole moment for the transition between levels $|i\rangle$ and $|j\rangle$, with $\widehat{e}_{L}$ being the unit polarization vector of the corresponding laser field. As shown in Fig. 1(c), all the light propagates along the $z$ axis within our SQW sample, and we consider a transverse magnetic polarized probe incident at an angle of $45^{\circ}$ with respect to the growth axis so that all transition dipole moments include a factor of $1 / \sqrt{2}$ as ISBTs are polarized along the growth axis. In the Schrödinger picture and in the rotating-wave approximation (RWA), the semiclassical Hamiltonian describing the system under study can be written as

$$
\begin{aligned}
H= & \sum_{j=1}^{4} E_{j}|j\rangle\langle j|-\hbar\left(k \Omega_{c} e^{-i \omega_{c} t}|3\rangle\left\langle 2\left|+\Omega_{c} e^{-i \omega_{c} t}\right| 4\right\rangle\langle 2|\right. \\
& \left.+q \Omega_{p} e^{-i \omega_{p} t}|3\rangle\left\langle 1\left|+\Omega_{p} e^{-i \omega_{p} t}\right| 4\right\rangle\langle 1|+\text { H.c. }\right) .
\end{aligned}
$$

Here $E_{j}=\hbar \omega_{j}$ is the energy of the subband level $|j\rangle(j=1,2,3,4)$, and the parameters $k=\mu_{32} / \mu_{42}, q=\mu_{31} / \mu_{41}$ represent the ratios between the dipole moments of the subband transitions. Working in the interaction picture, utilizing the RWA and the electric-dipole approximation (EDA), we derive the interaction Hamiltonian for the system as (assuming that $\hbar=1$ )

$$
\begin{aligned}
H_{I}= & \left(\Delta_{c}-\Delta_{p}\right)|2\rangle\left\langle 2\left|-\left(\Delta_{p}+\Delta\right)\right| 3\right\rangle\langle 3| \\
& +\left(\Delta-\Delta_{p}\right)|4\rangle\langle 4|+\left(\Omega_{c}|4\rangle\left\langle 2\left|+k \Omega_{c}\right| 3\right\rangle\langle 2|\right. \\
& \left.+\Omega_{p}|4\rangle\left\langle 1\left|+q \Omega_{p}\right| 3\right\rangle\langle 1|+\text { H.c. }\right),
\end{aligned}
$$

with $H_{0}=\left(\omega_{p}-\omega_{c}\right)|2\rangle\left\langle 2\left|+\omega_{p}\right| 3\right\rangle\left\langle 3\left|+\omega_{p}\right| 4\right\rangle\langle 4|, \Delta_{c}=\omega_{c}+\omega_{2}-\omega_{0}, \Delta_{p}=\omega_{p}-\omega_{0}, \omega_{0}=$ $\frac{1}{2}\left(\omega_{4}+\omega_{3}\right)$ and $\Delta=\left(E_{4}-E_{3}\right) / 2 . \Delta_{p}$ is the detuning between the frequency of the probe field and the average transition frequency $\omega_{0}$, and $\Delta_{c}$ is the detuning between the frequency of the strong-coupling field and the average frequency. It should be emphasized that the electron sheet density of the QW structure is small and the energy spacing between the subbands is large such that electron-electron effects have a very small influence on our results (for the contribution of the electronelectron scattering process to the the polarization dephasing rate, see the next paragraph). Besides, we have regarded $E_{1}$ as the energy origin. For simplicity, we assume that the Rabi frequency of the external fields $\Omega_{c}$ and $\Omega_{p}$ is real. Based on the equation of motion for the density operator in the interaction picture $(\partial \rho / \partial t=$ $\left.-i\left[H_{I}, \rho\right]+\Lambda \rho\right)$, we can easily derive the density matrix equations of motion, as follows:

$$
\begin{aligned}
\frac{\partial \rho_{44}}{\partial t}= & i \Omega_{c}\left(\rho_{24}-\rho_{42}\right)+i \Omega_{p}\left(\rho_{14}-\rho_{41}\right) \\
& -\left(2 \gamma_{41}+2 \gamma_{42}\right) \rho_{44}-\frac{\kappa}{2}\left(\rho_{34}+\rho_{43}\right), \\
\frac{\partial \rho_{33}}{\partial t}= & i k \Omega_{c}\left(\rho_{23}-\rho_{32}\right)+i q \Omega_{p}\left(\rho_{13}-\rho_{31}\right) \\
& -\left(2 \gamma_{31}+2 \gamma_{32}\right) \rho_{33}-\frac{\kappa}{2}\left(\rho_{34}+\rho_{43}\right),
\end{aligned}
$$




$$
\begin{aligned}
& \frac{\partial \rho_{22}}{\partial t}=i k \Omega_{c}\left(\rho_{32}-\rho_{23}\right)+i \Omega_{p}\left(\rho_{42}-\rho_{24}\right) \\
& -2 \gamma_{21} \rho_{22}+2 \gamma_{32} \rho_{33}+2 \gamma_{42} \rho_{44}, \\
& \frac{\partial \rho_{34}}{\partial t}=2 i \Delta \rho_{34}+i k \Omega_{c} \rho_{24}+i q \Omega_{p} \rho_{14}-i \Omega_{c} \rho_{32} \\
& -i \Omega_{p} \rho_{31}-\Gamma_{34} \rho_{34}-\frac{\kappa}{2}\left(\rho_{44}+\rho_{33}\right), \\
& \frac{\partial \rho_{24}}{\partial t}=i\left(\Delta-\Delta_{c}\right) \rho_{24}+i \Omega_{c}\left(\rho_{44}-\rho_{22}\right)+i k \Omega_{c} \rho_{34} \\
& -i \Omega_{p} \rho_{21}-\Gamma_{24} \rho_{24}-\frac{\kappa}{2} \rho_{23}, \\
& \frac{\partial \rho_{14}}{\partial t}=i\left(\Delta-\Delta_{p}\right) \rho_{14}+i \Omega_{p}\left(\rho_{44}-\rho_{11}\right)+i q \Omega_{p} \rho_{34} \\
& -i \Omega_{c} \rho_{12}-\Gamma_{14} \rho_{14}-\frac{\kappa}{2} \rho_{13}, \\
& \frac{\partial \rho_{23}}{\partial t}=-i\left(\Delta+\Delta_{c}\right) \rho_{23}-i q \Omega_{p} \rho_{21}+i \Omega_{c} \rho_{43} \\
& +i k \Omega_{c}\left(\rho_{33}-\rho_{22}\right)-\Gamma_{23} \rho_{23}-\frac{\kappa}{2} \rho_{13}, \\
& \frac{\partial \rho_{13}}{\partial t}=-i\left(\Delta+\Delta_{p}\right) \rho_{13}+i \Omega_{p} \rho_{43}-i k \Omega_{c} \rho_{12} \\
& +i q \Omega_{p}\left(\rho_{33}-\rho_{11}\right)-\Gamma_{13} \rho_{13}-\frac{\kappa}{2} \rho_{24}, \\
& \frac{\partial \rho_{12}}{\partial t}=i\left(\Delta_{c}-\Delta_{p}\right) \rho_{12}-i k \Omega_{c} \rho_{13}+i q \Omega_{p} \rho_{32} \\
& -i \Omega_{c} \rho_{14}+i \Omega_{p} \rho_{42}-\Gamma_{12} \rho_{12},
\end{aligned}
$$

together with $\rho_{i j}=\rho_{j i}^{*}$ and the carrier conservation condition $\rho_{11}+\rho_{22}+\rho_{33}+\rho_{44}=$ 1. Here the population decay rates $\gamma_{i l}$ (lifetime broadening), the dephasing decay rates $\gamma_{i j}^{\mathrm{dph}}$ and the polarization dephasing decay rates $\gamma_{i j}^{\mathrm{e}}$ - (dephasing linewidth corresponding to the respective transitions) are added phenomenologically in the above equations with the total decay rates (linewidth) being $\Gamma_{12}=\gamma_{12}^{\mathrm{dph}}+\gamma_{12}^{\mathrm{e}}-\frac{\gamma_{2 l}}{2}$, $\Gamma_{13}=\gamma_{13}^{\mathrm{dph}}+\gamma_{13}^{\mathrm{e}-\mathrm{e}}+\frac{\gamma_{3 l}}{2}, \Gamma_{14}=\gamma_{14}^{\mathrm{dph}}+\gamma_{14}^{\mathrm{e}-\mathrm{e}}+\frac{\gamma_{4 l}}{2}, \Gamma_{23}=\gamma_{23}^{\mathrm{dph}}+\gamma_{23}^{\mathrm{e}-\mathrm{e}}+\frac{\gamma_{2 l}+\gamma_{3 l}}{2}$, $\Gamma_{24}=\gamma_{24}^{\mathrm{dph}}+\gamma_{24}^{\mathrm{e}-\mathrm{e}}+\frac{\gamma_{2 l}+\gamma_{4 l}}{2}$, and $\Gamma_{34}=\gamma_{34}^{\mathrm{dph}}+\gamma_{34}^{\mathrm{e}-\mathrm{e}}+\frac{\gamma_{3 l}+\gamma_{4 l}}{2}$. Here $\gamma_{i l}$ is determined by longitudinal optical (LO) phonon emission events, at low temperature the $\gamma_{i j}^{\text {dph }}$ is determined by phonon scattering in our narrow doped well with monolayer fluctuation in the well width. ${ }^{17}$ The polarization dephasing rates of this structure $\gamma_{i j}^{\mathrm{e}-\mathrm{e}}$ are strongly dependent upon the electron-electron scattering process. The population decay rates can be calculated, for a certain temperature up to $10 \mathrm{~K}$, the carrier density is smaller than $10^{11} \mathrm{~cm}^{-2}$. In order to simplify the calculation, here the dephasing decay rates $\gamma_{i j}^{\mathrm{dph}}$ and $\gamma_{i j}^{\mathrm{e}-\mathrm{e}}$ are estimated according to Refs. 13 and 24 . Besides, the dephasing-type broadening $\kappa=\sqrt{\gamma_{3 l} \gamma_{4 l}}$ denotes the cross coupling of states $|4\rangle$ and $|3\rangle$ via the LO phonon decay. A more complete theoretical treatment 
taking into account this process for the dephasing rates is interesting but beyond the scope of this paper.

In the following analysis, we choose the parameters to be $\mu_{32}=\mu_{42}=\mu_{c}$ and $\mu_{31}=\mu_{41}=\mu_{p}$ (i.e. $k=1$ and $q=1$ ); and we take $\Gamma_{12}=0$ for the lifetime of levels $|2\rangle$ and $|1\rangle$ according to Ref. 36. By a straightforward semiclassical analysis, the above matrix elements can be used to calculate the total linear complex susceptibility $\chi$ of the probe transitions $|1\rangle \rightarrow|3\rangle$ and $|1\rangle \rightarrow|4\rangle$, i.e.

$$
\chi=\frac{N\left|\mu_{p}\right|^{2}\left(\rho_{13}+\rho_{14}\right)}{2 \hbar \varepsilon_{0} \Omega_{p}},
$$

where $N$ is the electron number density in the medium and $\varepsilon_{0}$ is the permittivity of free space. Based on Eq. (12), one can find that the gain-absorption coefficient for the probe field coupled to the transitions $|1\rangle \leftrightarrow|3\rangle(|4\rangle)$ is proportional to the term $\operatorname{Im}\left(\rho_{13}+\rho_{14}\right)$ in the limit of a weak probe field. $\operatorname{Im}\left(\rho_{13}+\rho_{14}\right)>0$ presents the absorption of the probe field; $\operatorname{Im}\left(\rho_{13}+\rho_{14}\right)<0$ presents the amplification of the probe field. In the following, we investigate the absorption properties of such a weak probe field for the transient- and steady-state processes. We begin by solving numerically the time-dependent differential equations (3)-(11) with the initial conditions $\rho_{11}(0)=1, \rho_{22}(0)=\rho_{33}(0)=\rho_{44}(0)=0$ and $\rho_{i j}(0)=0$ for $i \neq j$ $(i, j=1,2,3,4)$. Note that the parameters $\Omega_{c}, \Omega_{p}, \Delta_{c}, \Delta_{p}, \Delta, \gamma_{i j}$, and $\gamma_{i j}^{\mathrm{dph}}$ are defined as units of a free-will constant $\gamma$ in this paper.

\section{The Transient Absorption of the Probe Field}

Firstly, we examined the probe absorption under the transient-state process by using different sets of parameters.

We show in Fig. 2 the time evolution of the absorption coefficient $\operatorname{Im}\left(\rho_{13}+\rho_{14}\right)$ under the Raman resonant condition $\Delta_{c}=\Delta_{p}$ with the parameter values $\Omega_{p}=$

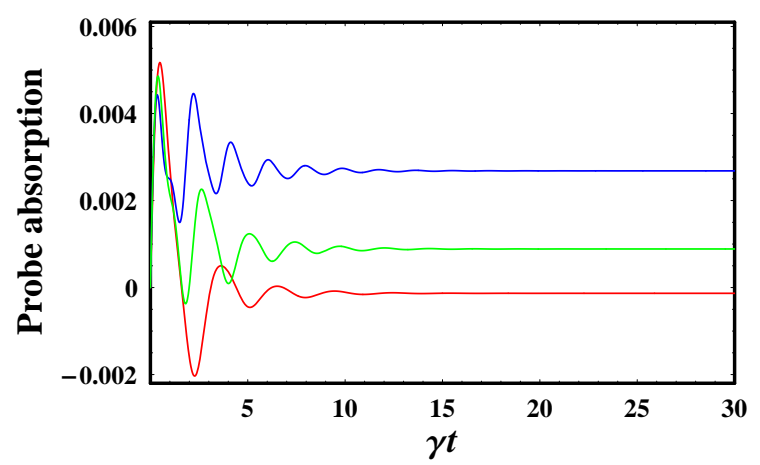

Fig. 2. (Color online) Transient absorption and amplification response for a probe laser field with different energy splitting between $|3\rangle$ and $|4\rangle, \Delta=4 \gamma$ (blue curve), $\Delta=2 \gamma$ (green curve), $\Delta=\gamma$ (red curve). Other fitting parameters are $\Omega_{p}=0.01 \gamma, \Delta_{c}=\Delta_{p}=0, \gamma_{3 l}=\gamma_{4 l}=0.4 \gamma$, and $\gamma_{i j}^{\mathrm{dph}}=\gamma_{i j}^{\mathrm{e}-\mathrm{e}}=0.1 \gamma$. 


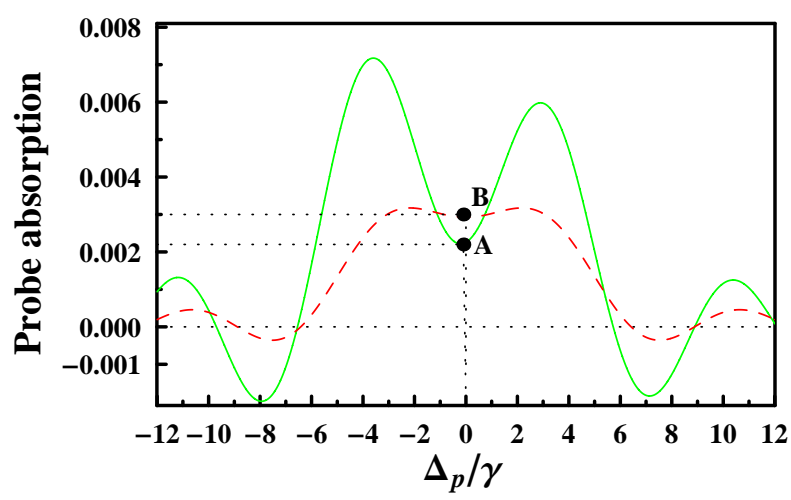

Fig. 3. (Color online) Transient absorption of the probe field as a function of dimensionless probe detuning $\Delta_{p} / \gamma$. Solid red curve: $\Delta=0$ corresponding to the three-level system. Dotted green curve: $\Delta=\gamma$ corresponding to the four-level system. Other fitting parameters used are $\Omega_{c}=2 \gamma, \Omega_{p}=0.01 \gamma, \Delta_{c}=0, \gamma_{3 l}=\gamma_{4 l}=0.4 \gamma$, and $\gamma_{i j}^{\mathrm{dph}}=\gamma_{i j}^{\mathrm{e}-\mathrm{e}}=0.1 \gamma$.

$0.01 \gamma, \Omega_{c}=4 \gamma, \Delta=\gamma, \gamma_{3 l}=\gamma_{4 l}=0.4 \gamma$, and $\gamma_{i j}^{\mathrm{dph}}=\gamma_{i j}^{\mathrm{e}-\mathrm{e}}=0.1 \gamma$. Here we have estimated that $\gamma_{i j}^{\mathrm{dph}}=\gamma_{i j}^{\mathrm{e}-\mathrm{e}}$ under this strong coupled field $\Omega_{c}=4 \gamma$ (the damping rates increase with the external field intensity but saturate in the limit $\left.\Omega_{c} \gg \Gamma_{23}, \Gamma_{24}\right) .{ }^{13}$ As shown in Fig. 1, with decreasing $\Delta$, the oscillatory frequency decreases, but the oscillatory amplitude increases. The probe field shows the oscillatory behavior versus the time before reaching the steady state, which is just like the period absorption and amplification in the high-density atomic system. The probe absorption increases rapidly to a maximum peak value, then it decreases gradually to a peak value and again increases with increasing evolution time, up to a steady-state value. Compared with the three-level EIT system, the transient behavior of the probe field can be tuned by adjusting $\Delta$ between the two upper levels $|3\rangle$ and $|4\rangle$. Figure 2 shows that the energy splitting $2 \Delta$ plays an important role in controlling the transient behavior of the probe field in such an asymmetric double QW system.

We show in Fig. 3 the magnitudes of the transient absorption of the probe field versus the probe detuning $\Delta_{p} / \gamma$. At the line center of the probe transition $\Delta_{p}=0$, the magnitude of the transient absorption of the probe field at point A corresponding to the splitting $2 \Delta$ is much smaller than the magnitude of the probe field at point B for the three-level EIT corresponding to $\Delta=0$. With appropriate values of parameters we obtain the absorption spectra shown in Fig. 4 for different intensities of the strong-coupling field. By increasing the intensity of the strong-coupling field, the absorption depth of the probe field becomes smaller accordingly, as expected for the coherent EIT. At the same time, with a larger intensity of the control field the transparency width increases, which corresponds to the splitting between the two upper levels $|3\rangle$ and $|4\rangle$. From the above analysis, we can conclude that the probe absorption at the line center $\left(\Delta_{p}=0\right)$ can be considerably suppressed due to the splitting $2 \Delta$. 


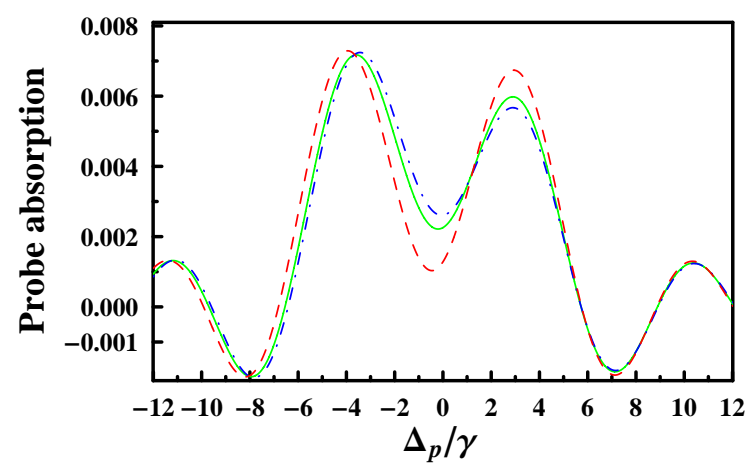

Fig. 4. (Color online) Transient absorption of the probe field as a function of dimensionless detuning $\Delta_{p} / \gamma$ for the four-level system with different strong coupling field $\Omega_{c}=1.5 \gamma$ (blue curve), $\Omega_{c}=2 \gamma$ (green curve) and $\Omega_{c}=1 \gamma$ (red curve). Other parameters are $\Omega_{p}=0.01 \gamma, \Delta=\gamma$, $\Delta_{c}=0, \gamma_{3 l}=\gamma_{4 l}=0.4 \gamma$, and $\gamma_{i j}^{\mathrm{dph}}=\gamma_{i j}^{\mathrm{e}-\mathrm{e}}=0.1 \gamma$.

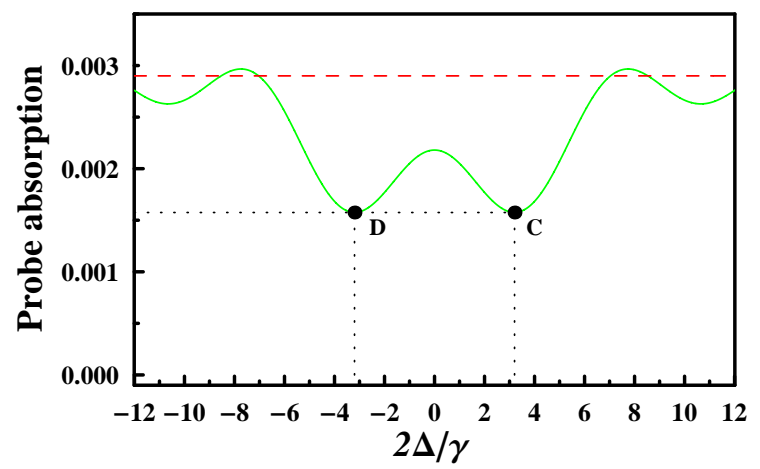

Fig. 5. (Color online) Transient absorption of the probe field as a function of dimensionless splitting $\Delta / \gamma$ between $|3\rangle$ and $|4\rangle$ (green curve). The dashed red line stands for the magnitude of the probe absorption for the three-level EIT at $\Delta_{p}=\Delta_{c}=0$. Other fitting parameters used are $\Delta_{p}=0, \Delta_{c}=0, \Omega_{p}=0.01 \gamma, \Omega_{c}=2 \gamma, \gamma_{3 l}=\gamma_{4 l}=0.4 \gamma$, and $\gamma_{i j}^{\mathrm{dph}}=\gamma_{i j}^{\mathrm{e}-\mathrm{e}}=0.1 \gamma$.

The calculated probe-absorption coefficient of such a weak probe laser versus the splitting $(2 \Delta)$ between $|3\rangle$ and $|4\rangle$ for the probe and coupling laser detunings $\Delta_{p}=\Delta_{c}=0$ is shown in Fig. 5 , in which the dashed red line stands for the magnitude of the probe absorption for the standard three-level EIT and the dotted green curve describes four-level with the positions $\mathrm{C}$ and $\mathrm{D}$ corresponding to the minimum of the probe absorption. We observe that the magnitude of the transient absorption of the probe field with the inclusion of the splitting $2 \Delta$ is always lower than the magnitude of the probe absorption with the three-level EIT at $\Delta_{p}=\Delta_{c}=$ 0. In Fig. 6, we plot absorption profiles versus the probe detuning $\Delta_{p}$ in the case of two-photon Raman resonance $\Delta_{p}=\Delta_{c}$. For this case, we find that the maximal absorption peak is always located below the the probe absorption of the standard three-level EIT. 


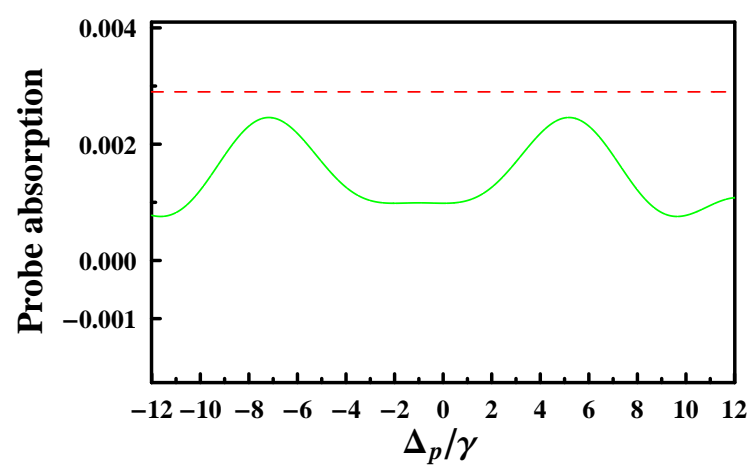

Fig. 6. (Color online) Transient absorption of the probe field as a function of dimensionless detuning $\Delta_{p} / \gamma$ for the Raman resonance $\Delta_{c}=\Delta_{p}$ (green curve). The dashed line stands for the magnitude of the probe absorption for three-level EIT at $\Delta_{c}=\Delta_{p}=0$. Other fitting parameters are $\Omega_{p}=0.01 \gamma, \Omega_{c}=2 \gamma, \Delta=\gamma, \gamma_{3 l}=\gamma_{4 l}=0.4 \gamma$, and $\gamma_{i j}^{\mathrm{dph}}=\gamma_{i j}^{\mathrm{e}-\mathrm{e}}=0.1 \gamma$.

\section{The Probe Absorption Under the Steady-State Condition}

Then we examined the probe absorption under the steady-state condition, i.e. $\partial \rho_{i j} / \partial t=0$. The general steady-state solutions to Eqs. (3)-(11) can be derived analytically (analyzing with two resonant coupling and probe fields), but the intricate and tedious expressions for the solutions corresponding to the situation of an off-resonant coupling field offer no clear physical sight. So we perform the numerical calculation here. The present process is most clearly understood according to the dressed states produced by the strong coupling field $\omega_{c}$ as shown in Fig. 1(b), i.e. the transitions $|2\rangle \leftrightarrow|3\rangle$ and $|2\rangle \leftrightarrow|4\rangle$ together with the coupling field treated as a total system forming the dressed states. Under the action of the strong coherent coupling field, levels $|3\rangle$ and $|4\rangle$ should be split into three dressed-state levels $| \pm\rangle$ and $|0\rangle$. The energy eigenstates of the dressed states for the $\Delta_{c}=0$ case are written as

$$
\begin{aligned}
|+\rangle & =-\frac{\Omega_{c}}{\sqrt{\Delta^{2}+2 \Omega_{c}^{2}}}|2\rangle-\frac{1}{2} \frac{\Delta-\sqrt{\Delta^{2}+2 \Omega_{c}^{2}}}{\sqrt{\Delta^{2}+2 \Omega_{c}^{2}}}|3\rangle+\frac{1}{2} \frac{\Delta+\sqrt{\Delta^{2}+2 \Omega_{c}^{2}}}{\sqrt{\Delta^{2}+2 \Omega_{c}^{2}}}|4\rangle, \\
|0\rangle & =\frac{\Delta}{\sqrt{\Delta^{2}+2 \Omega_{c}^{2}}}|2\rangle-\frac{\Omega_{c}}{\sqrt{\Delta^{2}+2 \Omega_{c}^{2}}}|3\rangle+\frac{\Omega_{c}}{\sqrt{\Delta^{2}+2 \Omega_{c}^{2}}}|4\rangle, \\
|-\rangle & =\frac{\Omega_{c}}{\sqrt{\Delta^{2}+2 \Omega_{c}^{2}}}|2\rangle+\frac{1}{2} \frac{\Delta+\sqrt{\Delta^{2}+2 \Omega_{c}^{2}}}{\sqrt{\Delta^{2}+2 \Omega_{c}^{2}}}|3\rangle-\frac{1}{2} \frac{\Delta-\sqrt{\Delta^{2}+2 \Omega_{c}^{2}}}{\sqrt{\Delta^{2}+2 \Omega_{c}^{2}}}|4\rangle .
\end{aligned}
$$

The corresponding energy eigenvalues are given by $\lambda_{0}=0$ and $\lambda_{ \pm}= \pm \sqrt{\Delta^{2}+2 \Omega_{c}^{2}}$. And the dressed-state transition dipole moments can be derived as $\mu_{+1}=\langle+|P| 1\rangle=$ $\mu_{p}, \mu_{01}=\langle 0|P| 1\rangle=0, \mu_{-1}=\langle-|P| 1\rangle=\mu_{p}$, where $P=\mu_{p}(|3\rangle\langle 1|+| 4\rangle\langle 1|)$ represents the polarization operator of the probe transition.

Based on the above analysis, it is straightforwardly shown that the transition between dressed levels $|0\rangle$ and $|1\rangle$ is forbidden due to the couplings of $|1\rangle \leftrightarrow|3\rangle$ 


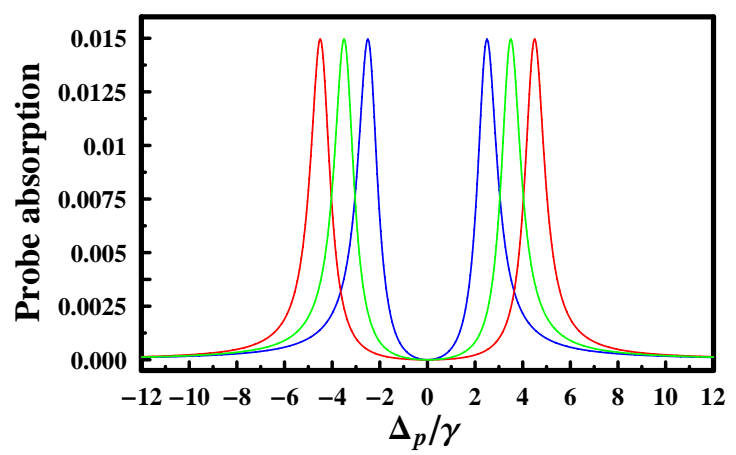

Fig. 7. (Color online) Imaginary part of the susceptibility of the probe transition as a function of dimensionless detuning $\Delta_{p} / \gamma$ under the condition of the steady-state process with $\Omega_{c}=2 \gamma$ (blue curve), $\Omega_{c}=3 \gamma$ (green curve) and $\Omega_{c}=4 \gamma$ (red curve). Other parameters used are $\Omega_{p}=0.01 \gamma$, $\Delta=\gamma, \Delta_{c}=0, \gamma_{3 l}=\gamma_{4 l}=0.4 \gamma$, and $\gamma_{i j}^{\mathrm{dph}}=\gamma_{i j}^{\mathrm{e}-\mathrm{e}}=0.1 \gamma$.

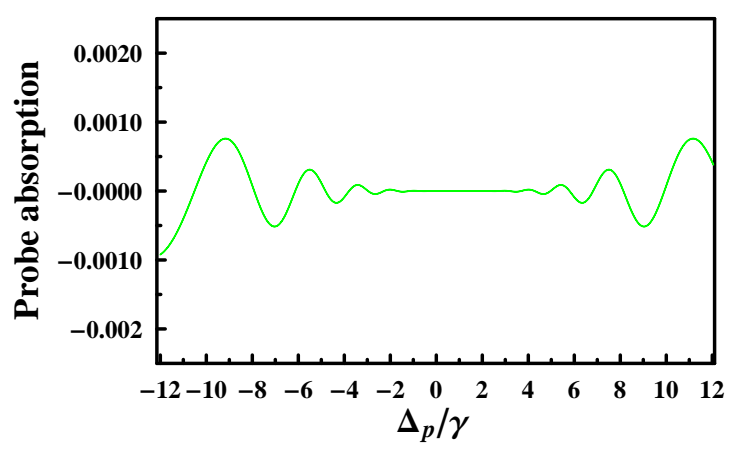

Fig. 8. Imaginary part of the susceptibility of the probe transition as a function of dimensionless detuning $\Delta_{p} / \gamma$ under the condition of the steady-state process for the Raman resonance $\left(\Delta_{c}=\right.$ $\left.\Delta_{p}\right)$ with the parameters being $\Omega_{p}=0.01 \gamma, \Omega_{c}=4 \gamma, \Delta=\gamma, \gamma_{3 l}=\gamma_{4 l}=0.4 \gamma$, and $\gamma_{i j}^{\mathrm{dph}}=$ $\gamma_{i j}^{\mathrm{e}-\mathrm{e}}=0.1 \gamma$

and $|1\rangle \leftrightarrow|4\rangle$. In Fig. 7, we can observe two symmetric absorption peaks with the same amplitude on the probe spectra in the case of $\Delta_{c}=0$ because of the relation $\mu_{+1}=\mu_{-1}$. The two absorption peaks correspond respectively to the dressed-state transitions $|1\rangle \leftrightarrow|+\rangle$ and $|1\rangle \leftrightarrow|-\rangle$, so the peaks are always located at $\Delta_{p}=$ $\pm \sqrt{\Delta^{2}+2 \Omega_{c}^{2}}$, and the difference between them becomes larger with increasing $\Omega_{c}$. Since the transition $|1\rangle \leftrightarrow|0\rangle$ in the dressed-state picture is canceled out by the interference and this interference always makes a negative contribution to the probe absorption, it is easy to understand that the probe absorption at the line center can be approximately equal to zero, i.e. the probe absorption can be completely suppressed, so that the absorption peak at the line center is too small to be observed. In Fig. 8, we plot the probe-absorption profile versus the probe detuning $\Delta_{p}$. One can find that the probe-absorption spectra are always approximately equal to zero within the range of the splitting $2 \Delta$ between two tunnel-coupled levels $(|3\rangle$ and $|4\rangle)$. 
This means that if the two-photon Raman resonance is satisfied $\left(\Delta_{p}=\Delta_{c}\right)$, we can observe the transparency window without requiring one- or two-photon detuning to exactly vanish.

\section{Discussions and Conclusion}

Because this focuses only on the low temperatures up to $10 \mathrm{~K}$, we have neglected the additional broadening in the above calculations and discussions. It is worth noting that the effects of additional broadening can be included by first rewriting the corresponding detunings, i.e. $\Delta_{p}=\omega_{p}-\omega_{0} \rightarrow \Delta_{p}-\Delta_{a 1}, \Delta_{c}=\omega_{c}+\omega_{2}-\omega_{0} \rightarrow$ $\Delta_{c}-\Delta_{a 2}$ with $\Delta_{a 1} \sim k_{p z}, \Delta_{a 2} k_{c z}$ being the additional broadening effects, which can be suppressed by a proper choice of the directions of the light. By consulting Ref. 37, we find that even for the moderate density, additional broadening effects are on the order of $1 \mathrm{meV}$. Combining the effect of the additional broadenings and the parameter values mentioned above, the influence of the probe absorption is only on the order $10^{-4}$. In addition, some other many-body effects may contribute the broadening effects, such as the depolarization effect, which renormalizes the freecarrier and carrier-field contributions. These contributions and their interplay have been investigated quite thoroughly by some authors in Refs. 38-41. Note that, due to the small carrier density considered here, these effects give only a small extent.

In conclusion, in the present paper, we have analyzed and discussed the probe absorption properties in a CQW under the conditions for the transient regime and the steady-state process by using direct numerical simulations based on the density matrix equations (3)-(11). Besides, we analytically showed the probe absorption mechanism for the steady-state process in the dressed-state picture. In the steadystate process, we showed that the probe absorption can be largely suppressed with the coupling field resonance $\left(\Delta_{c}=0\right)$, and completely eliminated under the condition of Raman resonance $\left(\Delta_{p}=\Delta_{c}\right)$ within the range of $2 \Delta$. In the transient-state process, we showed that the magnitude of the probe absorption at the line center of the probe transition $\Delta_{p}=0$ can be greatly suppressed. Compared with the EIT scheme in the three-level QW structures, ${ }^{12-14}$ our results show that the transparency hole is much broader due to the existence of the tunnel-coupling splitting $2 \Delta$. Besides, we did not require that the one- or two-photon detunings be exactly zero. As a result, the present investigation confirm the possibility of obtaining EIT in solids by using the asymmetric tunnel CQW. Our calculations also provide a guideline for the optimal design to achieve very fast and low-threshold all-optical switches in such semiconductor systems, which is much more practical than that in the atomic system because of its flexible design and the controllable coherent coupling strength.

Before ending, we note that we have used the one-dimensional model in the above analysis, and correspondingly the momentum-dependency of subband energies was ignored. In fact, there is no large discrepancy between the reduced one-dimensional calculation and the full two-dimensional calculation, and the related theoretical discussions can be found in Refs. 14 and 24 . 


\section{Acknowledgment}

This research is supported in part by the NSFC under Grant Nos. 10704017 and 10575040, by NFRPC 2005CB724508. We would like to thank Ite Yu for enlightening discussions.

\section{References}

1. S. E. Harris, Phys. Today 50 (1997) 36; M. Fleishhauer, A. Imamoglu and J. P. Marangos, Rev. Mod. Phys. 77 (2005) 633; M. D. Lukin, Rev. Mod. Phys. 75 (2003) 457; M. D. Lukin, P. Hemmer and M. O. Scully, Adv. At. Mol. Opt. Phys. 42 (2000) 347.

2. S. E. Harris, J. E. Field and A. Imamoglu, Phys. Rev. Lett. 64 (1990) 1107.

3. E. Arimondo, in Progress in Optics, ed. E. Wolf (Elsevier, Amsterdam, 1996), p. 257.

4. L. V. Hau et al., Nature 397 (1999) 594; C. Liu et al., ibid. 409 (2001) 490.

5. G. S. Agarwal and W. Harshawardhan, Phys. Rev. Lett. 77 (1996) 1039; Y. Li and M. Xiao, Opt. Lett. 21 (1996) 1064.

6. M. Yan, E. Rickey and Y. Zhu, Phys. Rev. A 64 (2001) 043807; M. Yan, E. Rickey and Y. Zhu, Opt. Lett. 26 (2001) 548.

7. Y. Wu and X. Yang, Phys. Rev. A 70 (2004) 053818; Y. Wu, ibid. 71 (2005) 053820; Y. Wu and L. Deng, Phys. Rev. Lett. 93 (2004) 143904; Y. Wu and X. Yang, Phys. Rev. A $\mathbf{7 1}$ (2005) 053806.

8. J. H. Wu et al., Opt. Lett. 28 (2003) 654.

9. S. E. Harris and Y. Yamamoto, Phys. Rev. Lett. 81 (1998) 3611.

10. Y. Wu, J. Saldana and Y. Zhu, Phys. Rev. A 67 (2003) 013811; ibid., Opt. Lett. 28 (2003) 631.

11. G. Alzetta, A. Gozzini, L. Moi and G. Orriols, Nuovo Cimento B 36 (1976) 5; E. Arimondo and G. Orriols, Lett. Nuovo Cimento D 17 (1976) 333.

12. D. E. Nikonov, A. Imamoglu and M. O. Scully, Phys. Rev. B 59 (1999) 12212.

13. S. M. Sadeghi, S. R. Leffler and J. Meyer, Phys. Rev. B 59 (1999) 15388; S. M. Sadeghi and J. Meyer, ibid. 61 (2000) 16841; S. M. Sadeghi and H. M. van Driel, ibid. 63 (2001) 045316.

14. L. Silvestri, F. Bassanil, G. Czajkowski and B. Davoudi, Eur. Phys. J. B 27 (2002) 89.

15. M. Phillips and H. Wang, Phys. Rev. Lett. 89 (2002) 186401.

16. M. Phillips and H. Wang, Opt. Lett. 28 (2003) 831; P. Ginzburg and M. Orenstein, Opt. Express. 14 (2006) 12467.

17. G. B. Serapiglia, E. Paspalakis, C. Sirtori, K. L. Vodopyanov and C. C. Phillips, Phys. Rev. Lett. 84 (2000) 1019.

18. J. F. Dynes, M. D. Frogley, M. Beck, J. Faist and C. C. Phillips, Phys. Rev. Lett. 94 (0000) 157403.

19. A. Imamoglu and R. J. Ram, Opt. Lett. 19 (1994) 1744; C.-R. Lee et al., Appl. Phys. Lett. 86 (2005) 201112.

20. A. A. Bastista and D. S. Citrin, Phys. Rev. Lett. 92 (2004) 127404; ibid., Phys. Rev. B 74 (2006) 195318.

21. E. Paspalakis, M. Tsaousidou and A. F. Terzis, Phys. Rev. B 73 (2006) 125344.

22. L. Tsang, D. Ahn and S. L. Chuang, Appl. Phys. Lett. 52 (1988) 697; E. Rosencher and Ph. Bois, Phys. Rev. B 44 (1991) 11315; H. Schmidt and A. Imamoglu, Opt. Commun. 131 (1996) 333. 
23. A. Joshi and M. Xiao, Appl. Phys. B 79 (2004) 65; H. O. Wijewardane and C. A. Ullrich, Appl. Phys. Lett. 84 (2004) 3984; J. H. Li, Phys. Rev. B 75 (2007) 155329.

24. H. Schmidt and R. J. Ram, Appl. Phys. Lett. 76 (2000) 3173; J. H. Wu, J. Y. Gao, J. H. Xu, L. Silvestri, M. Artoni, G. C. La Rocca and F. Bassani, Phys. Rev. Lett. 95 (2005) 057401; J. H. Wu, J. Y. Gao, J. H. Xu, L. Silvestri, M. Artoni, G. C. La Rocca and F. Bassani, Phys. Rev. A 73 (2006) 053818.

25. J. F. Dynes and E. Paspalakis, Phys. Rev. B 73 (2006) 233305.

26. J. F. Dynes, M. D. Frogley, J. Rodger and C. C. Phillips, Phys. Rev. B 72 (2005) 085323 .

27. S. M. Sadeghi, H. van Driel and J. M. Fraser, Phys. Rev. B 62 (2000) 15386.

28. E. Paspalakis, A. Kanaki and A. F. Terzis, Proc. SPIE 6582 (2007) 65821N.

29. J. Faist, F. Capasso, C. Sirtori, K. W. West and L. N. Pfeiffer, Nature 390 (1997) 589; H. Sun, S. Q. Gong, Y. P. Niu, S. Q. Jin, R. X. Li and Z. Z. Xu, Phys. Rev. B 74 (2006) 155314; W.-X. Yang, J. M. Hou and R. K. Lee, Phys. Rev. A 77 (2008) 033838 .

30. J. N. Heyman et al., Phys. Rev. Lett. 72 (1994) 2183; K. Craig et al., ibid. 76 (1996) 2382; B. Galdrikian and B. Birnir, ibid. 76 (1996) 3308; D. E. Nikonov et al., ibid. 79 (1997) 4633.

31. J. Z. Li and C. Z. Ning, Phys. Rev. Lett. 91 (2003) 097401; A. Olaya-Castro et al., Phys. Rev. B 68 (2003) 155305; C. W. Luo et al., Phys. Rev. Lett. 92 (2004) 047402; T. Muller et al., Phys. Rev. B $\mathbf{7 0}$ (2004) 155324.

32. F. T. Vasko and O. E. Raichev, Phys. Rev. B 51 (1995) 16965.

33. Y. Xue et al., Opt. Commun. 249 (2005) 231; J. H. Li, ibid. 274 (2007) 366.

34. H. G. Roskos et al., Phys. Rev. Lett. 68 (1992) 2216.

35. M. S. C. Luo et al., Phys. Rev. B 48 (1993) 11043; P. C. M. Planken et al., ibid. 48 (1993) 4903.

36. A. Neogi, H. Yoshida, T. Mozume and O. Wada, Opt. Commun. 159 (1999) 225.

37. I. Waldmüller, J. Förstner, S.-C. Lee, A. Knorr, M. Woerner, K. Reimann, R. A. Kaindl, T. Elsaesser, R. Hey and K. H. Ploog, Phys. Rev. B 69 (2004) 205307.

38. T. Shih, K. Reimann, M. Woerner, T. Elsaesser, I. Waldmüller, A. Knorr, R. Hey and K. H. Ploog, Phys. Rev. B 72 (2005) 195338.

39. R. J. Warburton, C. Gauer, A. Wixforth, J. P. Kotthaus, B. Brar and H. Kroemer, Phys. Rev. B 53 (1996) 7903; D. E. Nikonov, A. Imamoğlu, L. V. Butov and H. Schmidt, Phys. Rev. Lett. 79 (1997) 4633; B. Nottelmann, V. M. Axt and T. Kuhn, Physica B 272 (1999) 234.

40. S. V. Faleev and M. I. Stockman, Phys. Rev. B 66 (2002) 085318; I. Waldmüller, M. Woerner, J. Förstner and A. Knorr, Phys. Stat. Solidi B 238 (2003) 474.

41. S.-W. Chang, S.-L. Chuang, P.-C. Ku, C. J. Chang-Hasnian, P. Palinginis and H. Wang, Phys. Rev. B 70 (2004) 235333. 